\title{
COMPARACIÓN DE LAS PUNTUACIONES DEL SCL-90-R ENTRE PERSONAS CON DEPENDENCIA DE LA NICOTINA Y PERSONAS CON DEPENDENCIA DE LA COCAÍNA AL INICIO DEL TRATAMIENTO
}

\author{
ANA LÓPEZ, ELENA FERNÁNDEZ y ELISARDO BECOÑA \\ Universidad de Santiago de Compostela
}

\begin{abstract}
Resumen: La demanda de tratamiento por problemas con el consumo de sustancias está relacionada con la existencia de malestar en el sujeto. Dicho malestar, es con frecuencia producido por las consecuencias negativas derivadas del consumo de sustancias y la presencia de problemas psicopatológicos. El objetivo del estudio es comparar el malestar subjetivo percibido por el sujeto y las puntuaciones en las nueve escalas de síntomas psicopatológicos del Cuestionario de 90 Síntomas Revisado (SCL-90-R) en el momento de inicio del tratamiento, en personas con dependencia de la cocaína y personas con dependencia de la nicotina. La muestra está formada por 230 sujetos, 115 sujetos con dependencia de la cocaína y 115 con dependencia de la nicotina. Encontramos diferencias significativas entre el grupo con dependencia de la nicotina y el grupo con dependencia de la cocaína en todos los índices generales y en las subescalas de síntomas excepto somatización y ansiedad fóbica.
\end{abstract}

Palabras clave: dependencia, nicotina, cocaína, SCL-90-R.

\section{Comparison of the SCL-90-R score between nicotine dependence and cocaine dependence subjects at the beginning of treatment}

\begin{abstract}
The demand for treatment of problems on account of drug abuse is related to the presence of psychological distress. This distress is frequently produced by the negative consequences of drug abuse and the presence of psychopathological problems. The objective of the present study is to compare the psychological distress perceived by the subject with the score in the nine scales of psychopathological symptoms of the SCL-90-R at the start of treatment of subjects with cocaine dependence and subjects with nicotine dependence. The sample is formed by 230 subjects, 115 with cocaine dependence and 115 with nicotine dependence. We find significant differences between the group with nicotine dependence and the one with cocaine dependence in all the general indexes and in the subscales of symptoms except in somatization and phobic anxiety.
\end{abstract}

Keywords: dependence, nicotine, cocaine, SCL-90-R.

\section{INTRODUCCIÓN}

En los últimos años la evaluación de la presencia de problemas psicopatológicos en los consumidores de sustancias psicoactivas y en personas con otros problemas de adicción como

\section{Recibido 24 marzo 2008; aceptado 6 mayo 2008}

Correspondencia: Elisardo Becoña Iglesias, Universidad de Santiago de Compostela, Departamento de Psicología Clínica y Psicobiología, Campus Universitario Sur, 15782 Santiago de Compostela (España).

Correo-e: pcelisar@usc.es. la ludopatía o la adicción a móviles o internet está cobrando especial importancia, incluso su presencia en edades tempranas en las que con frecuencia empiezan estos problemas (Becoña, Vázquez y Míguez, 2001). La razón fundamental son las implicaciones que tiene en el tratamiento la presencia de estos problemas. Rosenthal y Westreich (1999) y Sánchez (2001), apuntan la necesidad de evaluar la presencia de síntomas psicopatológicos en todos los consumidores de sustancias que acuden a tratamiento, porque además de facilitar la definición del plan de in- 
tervención en el problema con el consumo de drogas, la evaluación nos facilita tener una visión global de la persona, más allá del consumo de drogas.

Una de las dificultades de realizar evaluación psicopatológica en los consumidores de sustancias, es la necesidad de contar con instrumentos adecuados para optimizar este proceso. No es posible administrar a todas las personas que demandan tratamiento una entrevista diagnóstica completa, tipo SCID-I (First, Spitzer, Gibbson, Williams y Smith-Benjamin, 1998) que evalúa todos los trastornos psicopatológicos del eje I, ya que el tiempo de administración es muy amplio y en estas personas hay también muchas otras áreas a evaluar (problemas legales, familiares, laborales, de salud física...).

Es necesario utilizar cuestionarios breves que detecten la presencia de síntomas psicopatológicos, los denominados cuestionarios de screening, como el SCL-90 (Derogatis, 1973) y su versión posterior el SCL-90-R (Derogatis, 1977). Se trata de un cuestionario adecuado para hacer una valoración inicial de la presencia de psicopatología a partir de nueve escalas de síntomas (Brown, Seraganian y Tremblay, 1994; Castaneda, Galanter y Franco, 1989; Landa, Fernández-Montalvo, López-Goñi y Lorea, 2006), y también evalúa el malestar subjetivo percibido por el sujeto (Derogatis, 2002). Este último aspecto es el que hace que este cuestionario sea también útil para realizar seguimientos, y determinar así la evolución del sujeto durante el tratamiento y tras la finalización del mismo, tal y como utilizaron Pedrero, Puerta, Segura y Martínez (2004) y Weiss, Mirin, Michael y Sollogub (1986).

Rose, Brown y Haertzen (1989) utilizaron el SCL-90-R para comparar a los consumidores de cocaína que demandan tratamiento con los que no lo demandan, respecto al índice de severidad global y a las medidas en las nueve dimensiones clínicas. Campbell y Stark (1990) utilizaron el SCL-90, junto a otros instrumentos, para determinar las diferencias entre sujetos con problemas de abuso en distintas sustancias respecto a la presencia de síntomas psicopatológicos. Kleinman, Miller, Millman, Woody, Todd, Kemp et al. (1990) y López y Be- coña (2006) lo utilizaron para evaluar la presencia de alteraciones en alguna de las nueve áreas, en una muestra de consumidores de cocaína cuando inician tratamiento. Y Kleinman, Kang, Lipton, Woody, Kemp y Millman (1992) utilizaron las puntuaciones obtenidas en el SCL90 como una de las variables para determinar la retención en el tratamiento de una muestra de sujetos con abuso de cocaína en tratamiento ambulatorio.

Brown et al. (1994) utilizaron el SCL-90-R para establecer diferencias entre las personas con dependencia del alcohol y de la cocaína en tratamiento, y las personas con dependencia sólo del alcohol. Consideran que es un instrumento que permite realizar una evaluación psicológica comprensiva en las nueve dimensiones clínicas que evalúa. Castaneda (1994) lo utilizó para evaluar los síntomas psicopatológicos en una muestra de consumidores de diversas sustancias, entre los que hay consumidores de cocaína, que tienen también otros problemas psicopatológicos.

El número de estudios que utilizan el SCL90-R en fumadores ha sido inferior. Destaca el estudio de Villar, Viñas, Ferrer, Caparrós, Pérez y Cornellá (2004) realizado en población universitaria para evaluar la relación entre malestar psicológico y fumar, y el de Moolchan, Radzius, Epstein, Gorelick, Cadet y Henningfield (2002) en el que analizan distintos instrumentos para evaluar la dependencia de la nicotina y su relación con el Índice General Sintomático del SCL-90R.

El objetivo del presente estudio es analizar las diferencias que existen entre un grupo de personas con dependencia de la cocaína y un grupo de personas con dependencia de la nicotina, en las distintas escalas de síntomas y en los índices generales del SCL-90-R administrado al inicio del tratamiento.

\section{MÉTODO}

\section{Participantes}

La muestra estaba formada por 230 personas, 152 hombres y 78 mujeres, que demandaron tratamiento por dependencia de la nicotina o de 
la cocaína. Participaron 115 personas que estaban en tratamiento por dependencia de la cocaína en seis centros públicos de drogodependencias de Galicia. Los criterios de inclusión fueron: la cocaína es la droga principal que motiva el tratamiento, dependencia de la cocaína según criterios del DSM-IV-TR, y llevar un mínimo de tres semanas y un máximo de seis semanas abstinente en el consumo de cocaína, para evitar la confusión de los síntomas del síndrome de abstinencia con los síntomas psicopatológicos. Los criterios de exclusión fueron: presencia de alteraciones psicóticas graves e incapacidad de responder a los instrumentos de evaluación.

En el caso de la dependencia de la nicotina son 115 personas con dependencia de la nicotina que demandaron tratamiento en la Unidad de Tabaquismo de la Universidad de Santiago de Compostela. Los criterios de inclusión fueron demandar tratamiento para dejar de fumar y tener dependencia de la nicotina evaluada con el Test de Fagerström (Heatherton, Kozlowski, Frecker y Fagerström, 1991) utilizando el punto de corte de seis (Fagerström, Kunze, Scoberberger, Breslau, Hughes, Hurt et al., 1996). Los criterios de exclusión fueron: presencia de alteraciones psicóticas graves e incapacidad de responder a los instrumentos de evaluación.

\section{Instrumentos de evaluación}

Entrevista Estructurada de DSM-IV (SCID) en su versión española (First et al., 1998), para evaluar la dependencia de la cocaína.

Test de Fageström de Dependencia de la Nicotina (Heatherton et al., 1991) para determinar la dependencia de la nicotina. Es un cuestionario de seis ítems, dos de ellos con cuatro opciones de respuesta y los cuatro restantes con dos opciones. La puntuación oscila entre cero y diez, siendo la interpretación la siguiente: entre cero y cinco, dependencia media o baja, y de seis en adelante, dependencia alta (Fagerström et al., 1996).

Cuestionario de 90 Sintomas Revisado (SCL90-R), en su versión española de Derogatis (2002). Evalúa el malestar subjetivo percibido por el sujeto a partir de 90 síntomas que se agrupan en nueve escalas (somatización, obsesióncompulsión, sensibilidad interpersonal, depresión, ansiedad, hostilidad, ansiedad fóbica, ideación paranoide y psicoticismo) y tres índices generales: Índice General Sintomático (IGS), Total de Síntomas Positivos (TSP) e Índice de Malestar (PSDI). El GSI evalúa la intensidad del sufrimiento psíquico y psicosomático global; el PST evalúa la amplitud y diversidad de la psicopatología a partir del número total de síntomas presentes; el PSDI indica la intensidad sintomática media, es decir, el estilo característico del individuo para experimentar el sufrimiento psíquico, si el sujeto maximiza o minimiza sus respuestas. El análisis de estos tres índices es uno de los aspectos más valorados de este cuestionario, ya que indican de forma general el malestar que tiene la persona. El SCL-90-R evalúa la existencia de tres niveles de morbilidad psiquiátrica: ausencia de morbilidad (población normal), presencia de morbilidad psiquiátrica menor (población temporo-mandibular), generalmente asociada a trastornos funcionales o psicosomáticos, y presencia de patología psiquiátrica que se puede enmarcar en un diagnóstico psiquiátrico específico (población psiquiátrica).

\section{Procedimiento}

En el caso de las personas con dependencia de la cocaína, fueron seleccionadas consecutivamente por los psicólogos de los Centros de Drogodependencias, entre septiembre de 2003 y abril de 2005. Si cumplían los criterios de inclusión en el estudio eran citados para ser evaluados por nosotros independientemente. Todos los sujetos firmaron el consentimiento informado para participar en el estudio. En el caso de las personas con dependencia de la nicotina, demandaron tratamiento en la Unidad de Tabaquismo de la Facultad de Psicología de la Universidad de Santiago de Compostela entre los años 2003 y 2007. La evaluación se realizó dentro del protocolo habitual de evaluación psicológica pre-tratamiento. Todos firmaron el consentimiento informado para participar en el estudio. 


\section{RESULTADOS}

Las características de la muestra que participó en el estudio las podemos ver en la Tabla 1. La mayor parte de la muestra son hombres $(66,1 \% ; n=152)$ y la edad media se sitúa en 37,51 años $(D T=10,55)$. En el grupo de personas con dependencia de la cocaína, el por- centaje de hombres $(86,1 \% ; n=99)$ es superior al de mujeres $(13,9 \% ; n=16)$. Mientras que en el caso de las personas con dependencia de la nicotina, el $46,1 \%$ son hombres $(n=53)$ y el $53,9 \%$ son mujeres $(n=62)$. La media de edad es superior en el grupo de nicotina: 44,09 años $(D T=9,75)$, frente a 30,9 años $(D T=6,41)$, del grupo de cocaína.

Tabla 1. Características de los sujetos que forman la muestra de personas con dependencia de la cocaína $(n=115)$ y de los sujetos con dependencia de la nicotina $(n=115)$

\begin{tabular}{|c|c|c|c|c|c|c|}
\hline & \multicolumn{4}{|c|}{ Sexo } & & \\
\hline & \multicolumn{2}{|c|}{ Hombres } & \multicolumn{2}{|c|}{ Mujeres } & \multicolumn{2}{|c|}{ Edad } \\
\hline & $n$ & $\%$ & $n$ & $\%$ & Media & $D T$ \\
\hline Personas con dependencia de la cocaína & 99 & 86,1 & 16 & 13,9 & 30,93 & 6,41 \\
\hline Personas con dependencia de la nicotina & 53 & 46,1 & 62 & 53,9 & 44,09 & 9,75 \\
\hline Total & 152 & 66,1 & 78 & 33,9 & 37,51 & 10,55 \\
\hline
\end{tabular}

Las puntuaciones medias en el SCL-90-R son superiores en el grupo de personas con dependencia de la cocaína en todas las subescalas de síntomas psicopatológicos, excepto en la escala de somatización. También tienen puntuaciones medias superiores a las obtenidas por el grupo con dependencia de la nicotina, en los tres índices generales. Hay diferencias significativas entre ambos grupos en la mayor parte de las escalas. En concreto, se han encontrado diferencias significativas entre ambos grupos para las siguientes variables del SCL-90-R: obsesión-compulsión, sensibilidad interpersonal, depresión, ansiedad, hostilidad, ideación paranoide, y psicoticismo (véase la Tabla 2).

Tabla 2. Diferencias en las puntuaciones medias en el SCL-90-R entre el grupo de personas con dependencia de la cocaína $(n=115)$ y el grupo de personas con dependencia de la nicotina $(n=115)$

\begin{tabular}{lrrrrc}
\hline & \multicolumn{2}{c}{ Cocaína } & \multicolumn{3}{c}{ Tabaco } \\
\cline { 2 - 5 } \multicolumn{1}{c}{ SCL-90-R } & Media & $D T$ & Media & DT & \multicolumn{1}{c}{$t$} \\
\hline Somatización & 0,66 & 0,63 & 0,80 & 0,76 & $-2,13^{*}$ \\
Obsesión-compulsión & 1,01 & 0,70 & 0,78 & 0,73 & $-4,15^{* * *}$ \\
Sensibilidad interpersonal & 0,87 & 0,73 & 0,51 & 0,60 & $-3,03^{* *}$ \\
Depresión & 1,03 & 0,78 & 0,73 & 0,72 & $-2,04^{*}$ \\
Ansiedad & 0,76 & 0,74 & 0,57 & 0,66 & $-3,62^{* * *}$ \\
Hostilidad & 0,65 & 0,65 & 0,37 & 0,53 & $-1,80$ \\
Ansiedad fóbica & 0,44 & 0,58 & 0,31 & 0,52 & $-4,45^{* * *}$ \\
Ideación paranoide & 1,00 & 0,76 & 0,57 & 0,72 & $-2,15^{*}$ \\
Psicoticismo & 0,65 & 0,65 & 0,46 & 0,89 & $-3,01^{* *}$ \\
Índice general sintomático & 0,83 & 0,56 & 0,60 & 0,57 & $-3,55^{* * *}$ \\
Total de síntomas positivos & 40,07 & 18,83 & 30,72 & 20,98 & $-0,59$ \\
Índice de malestar & 1,79 & 0,55 & 1,77 & 2,72 & - \\
\hline
\end{tabular}

$* p<0,05 ; * * p<0,01 ; * * * p<0,001$ 
Respecto a los niveles de morbilidad, en función de las tablas de contingencia a las que son asignadas los sujetos (normal/temporomandibular/psiquiátrica), también encontramos diferencias significativas entre ambos grupos $\left(\chi_{(1)}^{2}=13,458, p<0,01\right)$. Hay un mayor porcentaje de sujetos con dependencia de la nicotina $(n=62)$ que de sujetos con dependencia de la cocaína $(n=34)$ clasificados en las tablas que hacen referencia al grupo de población normal (53,0\% y $29,6 \%$, respectivamente), hay un menor porcentaje de sujetos con dependencia de la nicotina $(n=29)$ que de sujetos con dependencia de la cocaína $(n=39)$ clasificados en las tablas del grupo de población temporomandibular (25,2\% y $33,9 \%$, respectivamente), $\mathrm{y}$ hay un menor porcentaje de sujetos con dependencia de la nicotina $(n=25)$ que de suje- tos con dependencia de la cocaína $(n=42)$ clasificados en las tablas del grupo de población psiquiátrica $(21,7 \%$ y $36,5 \%$, respectivamente).

Si comparamos los datos obtenidos en el presente estudio con las puntuaciones medias de la población general y psiquiátrica utilizadas como baremo para la validación de este cuestionario en España (ver Figura 2), observamos que en el caso del grupo de dependencia de la cocaína las puntuaciones medias son superiores en todos los casos a las puntuaciones del grupo normativo normal, pero inferiores a las del grupo normativo psiquiátrico. Mientras que en el grupo de dependencia de la nicotina, no hay tanta diferencia con las medias de la población normal e incluso algunas son inferiores, como es el caso de la depresión $(0,68$ y 0,72 , respectivamente) y la hostilidad $(0,36$ y 0,45 , respectivamente).

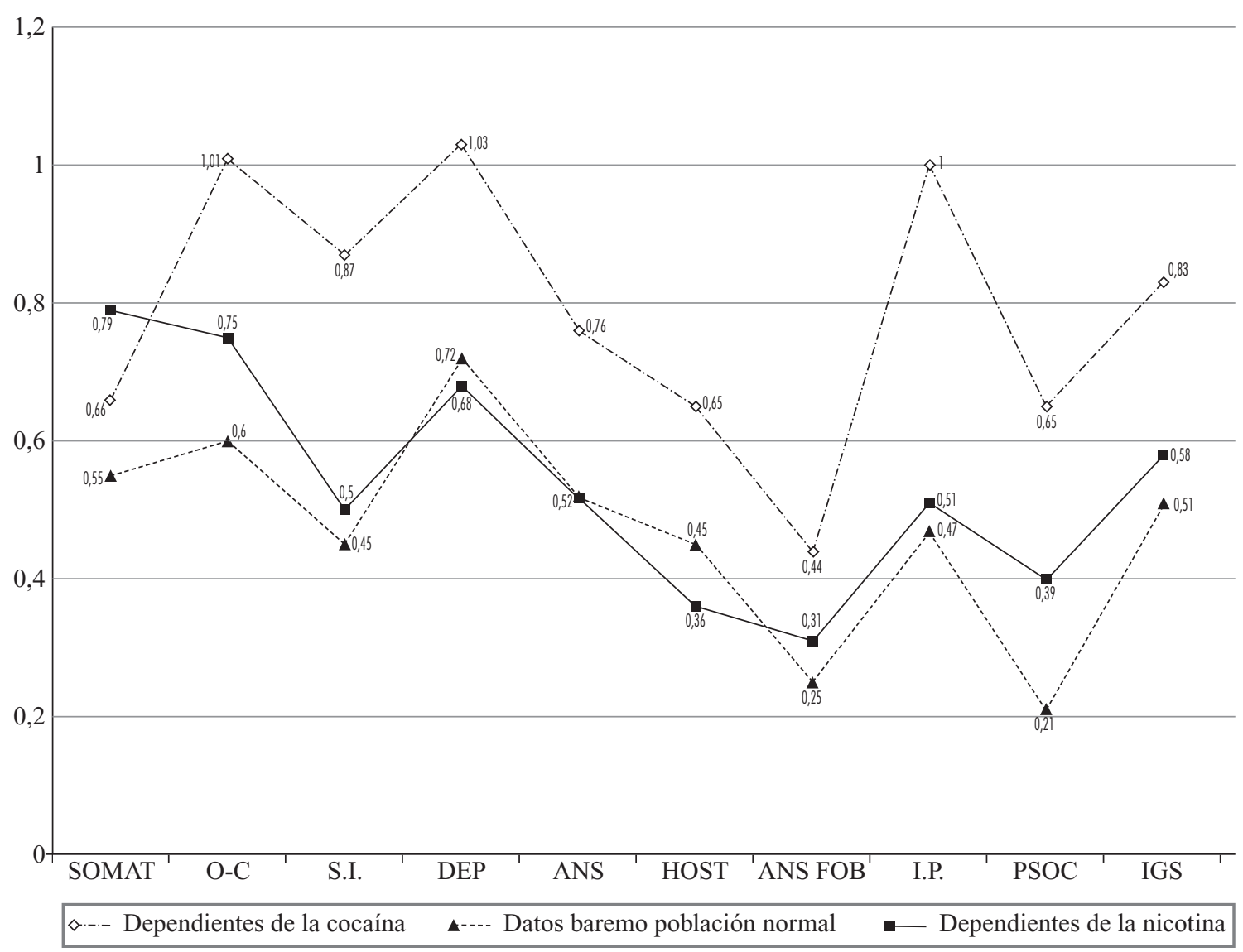

Figura 1. Comparación de las puntuaciones medias en el SCL-90-R de los grupos de dependencia de la cocaína $(n=115)$ y de la nicotina $(n=115)$, con los grupos normativos de la población normal y psiquiátrica.

Nota: SOMAT: somatización; O-C: obsesión-compulsión; S.I.= sensibilidad interpersonal; DEP = depresión; ANS = ansiedad; HOST: hostilidad; ANS FOB: ansiedad fóbica; I.P.: ideación paranoide; PSIC: psicoticismo; IGS: índice general sintomático. 


\section{DISCUSIÓN}

Las personas con dependencia de la cocaína que demandan tratamiento tienen puntuaciones significativamente superiores en la mayor parte de las subescalas de síntomas del SCL-90-R. Respecto a los tres índices generales, las puntuaciones de este grupo también son superiores, pero sólo hay diferencias estadísticamente significativas en el Índice General Sintomático y en el Total de Síntomas Positivos. Es decir, el grupo de cocaína tiene un mayor número de síntomas pero la intensidad de los mismos no es tan fuerte como para ser significativo respecto al grupo de tabaco.

Respecto a la clasificación de los sujetos en las tablas de morbilidad del SCL-90-R, también encontramos diferencias significativas entre ambos grupos: hay un mayor porcentaje de personas con dependencia de la nicotina clasificadas en la tabla de población normal, y hay un mayor porcentaje de personas con dependencia de la cocaína clasificadas en las tablas de temporo-mandibular y psiquiátrica.

Por lo tanto, las personas con dependencia de la cocaína cuando inician tratamiento presentan un mayor malestar subjetivo que las personas con dependencia de la nicotina. Dentro de las escalas de síntomas, en las que encontramos una mayor diferencia entre ambos grupos son las de sensibilidad-interpersonal, hostilidad e ideación paranoide. Estas escalas evalúan síntomas que con mucha frecuencia se asocian a los consumidores de cocaína (Ball, 1995; Kalayasiri, Kranzler, Weiss, Brady, Gueorguieva, Panhuysen et al., 2006; McCormick y Smith, 1995; Mooney, Sofuoglu, Dudish-Poulsen, y Hatsukami, 2006).

Las puntuaciones medias del grupo de nicotina son ligeramente superiores a las del grupo normativo de la población normal. Si comparamos los resultados de este grupo con los obtenidos en el estudio de Landa et al. (2006) con una muestra de personas con dependencia del alcohol en tratamiento, observamos que las puntuaciones del grupo de alcohol son superiores en todas las escalas excepto en obsesióncompulsión, sensibilidad interpersonal, ideación paranoide y el índice de malestar, en las cuales el grupo de nicotina tiene una puntuación media superior.

Las puntuaciones medias del grupo de cocaína son inferiores a las del grupo normativo de población psiquiátrica, siendo similares a las del grupo normativo temporo-mandibular. Por lo tanto, y tal y como define Derogatis (2002) este grupo normativo, es un grupo que tiene cierta morbilidad psicopatológica pero de tipo menor. No llega a considerarse población psiquiátrica.

Entre las limitaciones del uso de este cuestionario apuntar que está recomendado su uso como medida de malestar general (Bados, Balaguer y Coronas, 2005), ya que es altamente cuestionable interpretar las nueve dimensiones del SCL-90-R con fines clínicos.

A partir de los datos obtenidos podemos concluir que dos sustancias psicoactivas que producen una fuerte dependencia, la cocaína y la nicotina, producen distintos niveles de malestar en los sujetos que las consumen, en el momento en el que van a iniciar tratamiento.

Una posible explicación es que el tabaco, a pesar de las importantes consecuencias que tiene sobre la salud, sigue sin afectar de forma importante a otros ámbitos de la vida del sujeto como son el área laboral, económica o familiar, como también se encuentra con otros estudios con adolescentes (Bermúdez y Contreras, 2008). Mientras que el consumo de cocaína está muy relacionado con problemas familiares (cuando descubren el consumo), sociales (dejar de lado a los amigos no consumidores), económicos (debido al alto coste de la sustancia o al consumo cronificado) o laborales (no rendir lo suficiente, faltas continuas...). Claramente, en este caso, los consumidores de cocaína son menos resilientes (Becoña, 2006).

La realización de una evaluación previa al inicio del tratamiento es fundamental para la adaptación del mismo a las necesidades del sujeto. El SCL-90R es un cuestionario breve y sencillo de administrar e interpretar, que nos permite tener una primera visión del sujeto y la realización de posteriores seguimientos. Además, en la actualidad disponemos de una versión más abreviada de este cuestionario (Sandín, Valiente, Chorot, Santed, y Lostao, 2008) que puede facilitar su administración. 


\section{REFERENCIAS}

Bados, A., Balaguer, G., y Coronas, M. (2005). ¿Qué mide realmente el SCL-90R?: Estructura factorial en una muestra mixta. Psicología Conductual, 13, 181-196.

Ball, S. (1995). The Validity of an Alternative Five-Factor Measure of Personality in Cocaine Abusers. Psychological Assessment, 7, 148-154.

Becoña, E. (2006). Resiliencia: Definición, características y utilidad del concepto. Revista de Psicopatología y Psicología Clínica, 11, 125-146.

Becoña, E., Vázquez, F.L., y Míguez, M.C. (2001). Juego problema y sintomatología depresiva en adolescentes. Revista de Psicopatología y Psicología Clínica, 6, 1-16.

Bermúdez, J., y Contreras, A. (2008). Productores psicosociales del consumo de tabaco en adolescentes: Extensiones de la teoría de la conducta planificada. Revista de Psicopatología y Psicología Clínica, 13, 175186.

Brown, T.G., Seraganian, P., y Tremblay, J. (1994). Alcoholics also dependent on cocaine in treatment: do they different from «pure» alcoholics. Addictive Behaviors, 19, 105-112.

Castaneda, R., Galanter, M., y Franco, H. (1989). Selfmedication among addicts with primary psychiatric disorders. Comprehensive Psychiatry, 30, 80-83.

Campbell, B.K., y Stark, M.J. (1990). Psychopathology and personality characteristics in different forms of substance abuse. International Journal of the Addictions, 25, 1467-1474.

Derogatis, L.R. (1977). The SCL -90-R. Baltimore: Clinical Psychometric Research.

Derogatis, L.R. (2002). SCL-90-R. Cuestionario de 90 síntomas revisado. Manual. Madrid: TEA Ediciones.

Derogatis, L.R., Lipman, R.S., y Covi, L. (1973). The SCL90: An outpatient psychiatric rating scale. Psychopharmacology Bulletin, 9, 13-28.

Fagerström, K.O., Kunze, M., Scoberberger, R., Breslau, N., Hughes, J.R., Hurt, R.D., Puska, P., Ramstrom, L., y Zatonski, W. (1996). Nicotine dependence versus smoking prevalence: comparisons among countries and categories of smokers. Tobacco Control, 5, 52-56.

Heatherton, T.F., Kozlowski, L.T., Frecker, R.C., y Fagerström, K.O. (1991). The Fagerström Test for Nicotine Dependence: A revision of the Fagerström Tolerance Questionnaire. British Journal of Addictions, 86, 11191127.

Kalayasiri, R., Kranzler, H.R., Weiss, R., Brady, K., Gueorguieva, R., Panhuysen, C., Yang, B., Farrer, L., Gelernter, J., y Malison, R.T. (2006). Risk factors for cocaine-induced paranoia in cocaine-dependent sibling pairs. Drug and Alcohol Dependence, 84, 77-84.
Kleinman, P.H., Miller, A.B., Millman, R.B., Woody, G.E., Todd, T., Kemp, J., y Lipton, D.S. (1990). Psychopathology among cocaine abusers entering treatment. Journal of Nervous and Mental Disease, 178, 442-447.

Landa, N., Fernández-Montalvo, J., López-Goñi, J.J., y Lorea, I. (2006). Comorbilidad psicopatológica en el alcoholismo: un estudio descriptivo. Internacional Journal of Clinical and Health Psychology, 6, 253-269.

López, A., y Becoña, E. (2006). Dependencia de la cocaína y psicopatología a través del SCL-90-R. Revista de Psicopatología y Psicología Clínica, 11, 13-20.

McCormick, R.A., y Smith, M. (1995). Aggression and hostility in substance abusers: the relationship to abuse patterns, coping style, and relapse triggers. Addictive Behaviors, 20, 555-562.

Moolchan, E.T., Radzius, A., Epstein, D.H., Gorelick, D.A., Cadet, J.L., y Henningfield, J.E. (2002). The Fagerström test for nicotine dependence and the Diagnostic Interview Schedule: do they diagnose de same smokers? Addictive Behaviors, 27, 101-113.

Mooney, M., Sofuoglu, T.M., Dudish-Poulsen, S., y Hatsukami, D.K. (2006). Preliminary observations of paranoia in a human laboratory study of cocaine. Addictive Behaviors, 31, 1245-1251.

Pedrero, E.J., Puerta, C., Segura, I., y Martínez, S. (2004). Evolución de la sintomatología psicopatológica de los drogodependientes a lo largo del tratamiento. Trastornos Adictivos, 6, 175-191.

Rose, M.R., Brown, B.S., y Haertzen, Ch.A. (1989). Comparison of the characteristics and functioning of cocaine treatment and cocaine research subjects. American Journal of Drug and Alcohol Abuse, 15, 251-260.

Rosenthal, R.N., y Westreich, L. (1999). Treatment of persons with diagnoses of substance use disorders and other psychological problems. En B.S. McCrady y E.E. Epstein (Eds.), Addictions. A comprehensive guidebook (pp.105-120). New York: Oxford University Press.

Sánchez, E. (2001). Trastornos adictivos y otros trastornos mentales. Psiquiatría Biológica, 8, 64-73.

Sandín, B., Valiente, R.M., Chorot, P., Santed, M.A., y Lostao, L. (2008). SA-45: Forma abreviada del SCL90. Psicothema, 20, 290-296.

Spitzer, R.L., Endicott, J., y Robins, E. (1978). Research Diagnostic Criteria: rationale and reliability. Archives of General Psychiatry, 35, 773-785.

Villar, E., Viñas, F., Juan, J., Caparrós, B., Pérez, I., y Cornellá, M. (2004). Dimensiones psicopatológicas asociadas al consumo de tabaco en población universitaria. Anales de Psicología, 20, 33-46.

Weiss, R.D., Mirin, S.T., Michael, J.L., y Sollogub, A.C. (1986). Psychopathology in chronic cocaine abusers. American Journal of Drug and Alcohol Abuse, 12, 17-29. 